\title{
Irreversible effects of anticholinergic withdrawal in the elderly: a case report
}

\author{
Charisse Chehovich $^{\mathrm{a},{ }^{*}}$, Claudia Lee ${ }^{\mathrm{a}}$, Tammie Lee Demler ${ }^{\mathrm{a}, \mathrm{b}, \mathrm{c}}$ \\ ${ }^{a}$ Office of Mental Health, Buffalo Psychiatric Center, New York, USA. \\ ${ }^{b}$ School of Pharmacy and Pharmaceutical Sciences, University at Buffalo, New York, USA. \\ ${ }^{c}$ Department of Psychiatry, School of Medicine, University at Buffalo, New York, USA.
}

\begin{abstract}
Anticholinergics, such as benztropine and trihexyphenidyl, are a class of medications that have been used to treat several different conditions including antipsychotic-induced extrapyramidal side effects (EPS) that are most often associated with first-generation antipsychotics (FGAs), such as haloperidol and fluphenazine. Many other medications, including antimuscarinics, antipsychotics, and antidepressants, also have anticholinergic effects. In this report, we review the case of an 80 -year-old male who experiences irreversible anticholinergic withdrawal effects following the discontinuation of trihexyphenidyl and trospium secondary to side effects. Discontinuation of anticholinergics must be approached with care as abrupt withdrawal can lead to cholinergic rebound and muscular rigidity, and in some cases can lead to acute hospitalization and an inability to return to baseline functioning, as seen in our elderly patient.

Keywords: Anticholinergic withdrawal, trihexyphenidyl, trihexyphenidyl withdrawal, trospium, anticholinergic drugs, cholinergic rebound
\end{abstract}

\section{Introduction}

Anticholinergic medications can be used for many disease states, including irritable bowel syndrome, Parkinson's disease, peptic ulcer disease, urinary incontinence, and for the prophylaxis and treatment of drug-induced extrapyramidal effects secondary to antipsychotic use [1-3]. While anticholinergics can clinically benefit certain conditions, they are not without risk. Due to their mechanism of action on the central and peripheral nervous systems to induce their effects, anticholinergic medications can also have serious side effects ranging from constipation and dry mouth to cognitive impairment, hallucinations, tachyarrhythmias, and congestive heart failure [1]. Trihexyphenidyl and trospium are two such anticholinergic medications. Trihexyphenidyl is a medication considered to have a "high anticholinergic load", and was first approved by the Food and Drug Administration (FDA) in

\footnotetext{
* Corresponding author: Charisse Chehovich

Mailing address: Office of Mental Health, Buffalo Psychiatric Center, New York, USA.

Email: Charisse.Chehovich@omh.ny.gov

Received: 11 March 2021 / Accepted: 08 June 2021
}

April 2003 and is indicated for the treatment of Parkinson's Disease and drug-induced extrapyramidal effects, especially in those treated with a FGA, such as fluphenazine $[1,2,4]$. Trospium on the other hand is used to treat overactive bladder and has low blood brain barrier penetration due to its hydrophilic properties. For this reason, trospium does not exert anticholinergic activity on the central nervous system [5]. The risks associated with anticholinergic medications can lead to discontinuation, but while there is much discussion regarding anticholinergic effects and burden, providers must also be aware of the consequences of abrupt anticholinergic discontinuation leading to withdrawal. Anticholinergic withdrawal can lead to cholinergic rebound which includes urinary urgency, sweating, and nausea [6]. According to Saheed and colleagues, the most common symptom of trihexyphenidyl withdrawal was rigidity [7]. In other cases, anticholinergic withdrawal can be so severe it leads to hospitalization, as noted in this case report.

\section{Case report}

An 80-year-old black male was admitted to an inpatient psychiatric facility in July 2016 after he was found wandering the halls of his apartment building carrying a knife, as he believed someone was trying to murder him. He 
began showing signs of mental illness at the age of thirty and was hospitalized on multiple occasions within inpatient psychiatric settings. As of 2019, he had a psychiatric diagnosis of schizophrenia treated with fluphenazine decanoate and medical diagnoses to include constipation treated with polyethylene glycol, hyperlipidemia treated with atorvastatin, hypertension treated with amlodipine, prostate cancer treated with monthly leuprolide injections, vitamin D deficiency treated with cholecalciferol, glaucoma treated with latanoprost, dry eyes treated with polyvinyl alcohol, gastroesophageal reflux disease (GERD) treated with omeprazole, osteoarthritis treated with acetaminophen and menthol/methyl salicylate cream, and urinary incontinence treated with tamsulosin and trospium. The patient also received trihexyphenidyl for prevention of extrapyramidal side effects (EPS) (Table 1).

Coadministration of fluphenazine with leuprolide can decrease the effectiveness of leuprolide in addition to increasing the risk of QTc prolongation. However, the cardiovascular events associated with leuprolide may be due to hypogonadism rather than leuprolide itself [8]. As the patient was stabilized on fluphenazine prior to initiation of leuprolide and fluphenazine does not have a high risk of QTc prolongation, the decision to continue fluphenazine outweighed the risk of QTc prolongation, and the patient's cardiac function was monitored [9]. Fluphenazine in addition to trospium and trihexyphenidyl can increase the risk of anticholinergic effects, especially in the elderly. Alternative therapies that result in a lower anticholinergic burden should be considered when possible. Mirabegron is a $\beta 3$-adrenoceptor agonist used for the treatment of overactive bladder and does not have anticholinergic properties [10]. Mirabegron is currently only available as a brandnamed product, and its expense could limit the use of this medication as other generic alternatives are on the market. Due to the patient's inpatient status, this combination of fluphenazine, trospium, and trihexyphenidyl was deemed appropriate as the patient could be monitored around the clock for signs of anticholinergic effects. Calcium channel blockers, such as amlodipine can increase the risk of hypotensive effects in combination with tamsulosin. It is important to monitor a patient's blood pressure on a regular basis. Based on all factors, the patient's clinical team concluded that the benefits of using this combination of medications outweighed the risk of potential drug interactions because he could be monitored in the inpatient setting. To mitigate the risk of adverse drug reactions, clinicians should prescribe as few medications as are deemed necessary for treatment. When alternative safer therapy is available, it should be considered.

In May of 2019, the patient began complaining of difficulty with urination and trospium was discontinued. Shortly after, a foley catheter was inserted on June $25^{\text {th }}$ and due to its potential anticholinergic effects leading to urinary retention, trihexyphenidyl was discontinued on June $26^{\text {th }}$. The foley catheter was removed shortly after, on June $28^{\text {th }}$, with continuous output monitoring. On July $1^{\text {st }}$, the patient complained of fatigue and was noted to be having difficulty ambulating, increased drooling and difficulty using hands effectively to use a urinal and hold a cup. The

Table 1. Inpatient Medication List

\begin{tabular}{|c|c|c|c|c|c|}
\hline Medication & Dose & Directions & Indication & $\begin{array}{c}\text { Date of } \\
\text { Initiation }\end{array}$ & $\begin{array}{c}\text { Date of } \\
\text { Discontinuation }\end{array}$ \\
\hline Acetaminophen & $325 \mathrm{mg}$ & $\begin{array}{l}650 \mathrm{mg} \text { by mouth twice daily for pain } \\
\text { and every } 4 \text { hours as needed }\end{array}$ & Osteoarthritis pain & $9 / 18 / 2018$ & N/A \\
\hline Amlodipine & $5 \mathrm{mg}$ & $5 \mathrm{mg}$ by mouth daily & Hypertension & $7 / 26 / 2016$ & N/A \\
\hline Atorvastatin & $20 \mathrm{mg}$ & $20 \mathrm{mg}$ by mouth at bedtime & Hyperlipidemia & $1 / 24 / 2017$ & N/A \\
\hline Cholecalciferol & $10 \mathrm{mcg}$ & 20 mcg by mouth daily & Vitamin D deficiency & $8 / 7 / 2016$ & N/A \\
\hline $\begin{array}{l}\text { Fluphenazine } \\
\text { decanoate }\end{array}$ & $37.5 \mathrm{mg}$ & $37.5 \mathrm{mg}$ intramuscularly every other week & Psychosis & $3 / 21 / 2018$ & N/A \\
\hline Latanoprost eye drops & $0.005 \%$ & One drop into each eye at bedtime & Glaucoma & $10 / 10 / 2018$ & N/A \\
\hline $\begin{array}{l}\text { Menthol/ methyl } \\
\text { salicylate cream }\end{array}$ & $10 \% / 30 \%$ & $\begin{array}{l}\text { Apply a small amount four times daily } \\
\text { as needed }\end{array}$ & Osteoarthritis pain & $3 / 27 / 2018$ & N/A \\
\hline Omeprazole & $20 \mathrm{mg}$ & $20 \mathrm{mg}$ by mouth daily & GERD* & $8 / 24 / 2017$ & $\mathrm{~N} / \mathrm{A}$ \\
\hline Polyethylene glycol & 17 grams & 17 grams by mouth daily & Constipation & $8 / 29 / 2018$ & $\mathrm{~N} / \mathrm{A}$ \\
\hline $\begin{array}{l}\text { Polyvinyl alcohol eye } \\
\text { drops }\end{array}$ & $1.4 \%$ & One drop into each eye twice daily & Dry eyes & $10 / 10 / 2018$ & N/A \\
\hline Tamsulosin & $0.4 \mathrm{mg}$ & $0.4 \mathrm{mg}$ by mouth twice daily & Urinary incontinence & $3 / 31 / 2017$ & N/A \\
\hline Trihexyphenidyl & $2 \mathrm{mg}$ & $2 \mathrm{mg}$ by mouth twice daily & EPS* prevention & $7 / 11 / 2018$ & $6 / 26 / 2019$ \\
\hline Trospium & $20 \mathrm{mg}$ & $20 \mathrm{mg}$ by mouth at bedtime & Urinary incontinence & $6 / 13 / 2017$ & $6 / 12 / 2019$ \\
\hline
\end{tabular}

*GERD: gastroesophageal reflux disorder, EPS: extrapyramidal side effects 
patient was sent for further evaluation to rule out a cerebrovascular event on July $2^{\text {nd }}$ due to difficulty swallowing and moving his legs. He was admitted for hospitalization for further treatment and evaluation. An MRI of the brain with and without contrast showed a tiny right sided lacunar infarct involving the posterior limb of the right internal capsule with severe periventricular/subcortical deep white matter microangiopathy, but this was not thought to account for his symptoms as the lacunar infarct could not have resulted in the severity of the symptoms. Neuroleptic malignant syndrome (NMS) was also considered, but ultimately determined to not be a factor in the absence of a pyrexia or an elevated creatine phosphokinase (CPK). Additionally, a review of the patient's medications did not indicate that any of the remaining medications would have influenced the severity of the patient's symptoms.

On July $10^{\text {th }}$, the patient was unable to stand independently, ambulate, or feed himself due to muscle weakness. It was concluded that the patient was experiencing withdrawal of anticholinergics leading to generalized weakness. Trihexyphenidyl was reintroduced at half the discontinuation dose, $1 \mathrm{mg}$ twice daily. The patient was transferred to subacute rehab on July $12^{\text {th }}$. As of August 30 th, the patient did not have much improvement in walking and eating. It was determined that placement back into his inpatient psychiatric facility was not appropriate due to the level of care he now required.

Prior to transfer to an acute medical care hospital, the patient was independent with activities of daily living. While admitted to acute care, he required assistance for functional mobility, ambulation, and activities of daily living. Despite reintroduction of trihexyphenidyl and rehabilitation, the patient did not return to baseline and did not regain his strength and was placed in a skilled nursing facility.

\section{Discussion}

It has been estimated that $22-37 \%$ of adults at least 60 years of age are prescribed at least one anticholinergic medication [11]. In patients on depot FGAs, 32.8\% can experience EPS, which can lead to a prescription for an anticholinergic to treat this side effect [12]. Adults over 65 years of age are more likely to have a higher anticholinergic burden than the rest of the population [13]. There are several studies that discuss the potential anticholinergic side effects experienced by elderly patients [6,14-16]. With increased age, there is greater decline in production of acetylcholine which can lead to an increased risk of side effects in older populations [17]. Side effects can include dizziness, blurred vision, drowsiness, cognitive effects, tachycardia, and as our patient experienced, urinary retention [18].

Given the risk of side effects in elderly populations, anticholinergic discontinuation could be considered beneficial based on a clinical risk versus benefit analysis. However, discontinuation, particularly abrupt discontinuation, does not come without its own risks [16]. Anxiety, nau- sea, sweating, urinary incontinence, and tachycardia are withdrawal effects associated with cholinergic rebound syndrome due to anticholinergic discontinuation [19]. Trihexyphenidyl can have a higher risk of anticholinergic discontinuation syndrome due to its high affinity for muscarinic receptors, as anticholinergics exert their therapeutic effects via muscarinic acetylcholine receptor antagonism [20].

To decrease this risk of these adverse effects, many recommend tapering of anticholinergics rather than abrupt discontinuation $[3,19]$. While there are no guidelines to indicate an appropriate anticholinergic taper, one recommendation is to decrease the dose by $2-5 \mathrm{mg}$ equivalents of trihexyphenidyl every six weeks [21]. Based on these recommendations, it would have been most appropriate to decrease the dose of trihexyphenidyl from $2 \mathrm{mg}$ twice daily to $2 \mathrm{mg}$ once daily for six weeks, and then discontinue the dose thereafter for our patient. Regarding trospium discontinuation, there is no taper schedule available. The patient was on trospium $20 \mathrm{mg}$ once daily at bedtime, and an abrupt discontinuation would have been appropriate based on dose. However, due to age and risk of withdrawal due to the discontinuation of multiple anticholinergic agents, a taper schedule may have been beneficial based on patient specific factors. Unfortunately, the 80 -year-old patient's onset of urinary retention resulted in a more urgent treatment intervention.

Abrupt anticholinergic withdrawal can lead to many side effects including a marked irreversible change in functioning, especially in the elderly as noted in this case report. More studies should be conducted to detect a difference in the risk of withdrawal of anticholinergic medications between young and old patients. However, it can be inferred that elderly patients may be at a greater risk of anticholinergic withdrawal effects due to their higher anticholinergic burden and changes in pharmacodynamics and pharmacokinetics with age. In clinical practice, anticholinergics should be slowly tapered due to the risk of potential lasting effects, which can be reduced or completely avoided with a gradual discontinuation. Tapering, rather than abruptly discontinuing, medication can avoid or minimize medication discontinuation effects [19]. Providers should be aware of potential discontinuation effects with anticholinergic medications, and anticholinergic burden should be considered when deciding if it is appropriate to taper or discontinue an anticholinergic medication [6].

\section{Declarations}

Authors' contributions: Wrote manuscript with support and contributions from other authors: Chehovich C; provided support and contributions to the manuscript: Lee $\mathrm{C}$, Demler TL.

Conflict of interest: All authors declared that there are no conflicts of interest. 


\section{References}

1. López-Álvarez J, Sevilla-Llewellyn-Jones J, Agüera-Ortiz L. Anticholinergic drugs in geriatric psychopharmacology. Frontiers in neuroscience, 2019, 13: 1309.

2. Sheikh W A. Prophylactic use of trihexyphenidyl (artane) in schizophrenia and psychosis: a critical review of literature to guide for evidence based practice in Zambia. Medical Journal of Zambia, 2019, 46(2): 133-139.

3. Desmarais J E, Beauclair L, Annable L, et al. Effects of discontinuing anticholinergic treatment on movement disorders, cognition and psychopathology in patients with schizophrenia. Therapeutic advances in psychopharmacology, 2014, 4(6): 257-267.

4. Brocks D R. Anticholinergic drugs used in Parkinson's disease: an overlooked class of drugs from a pharmacokinetic perspective. J Pharm Pharm Sci, 1999, 2(2): 3946.

5. Chancellor M, Boone T. Anticholinergics for overactive bladder therapy: central nervous system effects. CNS neuroscience \& therapeutics, 2012, 18(2): 167-174.

6. Gnjidic D, Hilmer S, Bell J, et al. Anticholinergic burden: Considerations for older adults. 2017.

7. Olanrewaju R S, Mannir A. Trihexyphenidyl abuse in psychiatric outpatient clinic of a general hospital in Northern Nigeria. Journal of Addiction Medicine and Therapeutic Science, 2020, 6(1): 003-008.

8. Smith M R, Klotz L, Persson B E, et al. Cardiovascular safety of degarelix: results from a 12-month, comparative, randomized, open label, parallel group phase III trial in patients with prostate cancer. The Journal of urology, 2010, 184(6): 2313-2319.

9. Beach S R, Celano C M, Noseworthy P A, et al. QTc prolongation, torsades de pointes, and psychotropic medications. Psychosomatics, 2013, 54(1): 1-13.

10. Andersson K E. On the site and mechanism of action of $\beta 3$-adrenoceptor agonists in the bladder. International neurourology journal, 2017, 21(1): 6 .

11. Sittironnarit G, Ames D, Bush A I, et al. Effects of anticholinergic drugs on cognitive function in older Australians: results from the AIBL study. Dementia and geriatric cog- nitive disorders, 2011, 31(3): 173-178.

12. Novick D, Haro J M, Bertsch J, et al. Incidence of extrapyramidal symptoms and tardive dyskinesia in schizophrenia: thirty-six-month results from the European schizophrenia outpatient health outcomes study. Journal of clinical psychopharmacology, 2010, 30(5): 531-540.

13. Lipovec N C, Jazbar J, Kos M. Anticholinergic burden in children, adults and older adults in Slovenia: A Nationwide database study. Scientific Reports, 2020, 10(1): 1-8.

14. Carrière I, Fourrier-Reglat A, Dartigues J F, et al. Drugs with anticholinergic properties, cognitive decline, and dementia in an elderly general population: the 3-city study. Archives of internal medicine, 2009, 169(14): 1317-1324.

15. Heinik J. Effects of trihexyphenidyl on MMSE and CAMCOG scores of medicated elderly patients with schizophrenia. International psychogeriatrics, 1998, 10(1): 103-108.

16. Nishtala P S, Salahudeen M S, Hilmer S N. Anticholinergics: theoretical and clinical overview. Expert opinion on drug safety, 2016, 15(6): 753-768.

17. Hsu W H, Wen Y W, Chen L K, et al. Comparative associations between measures of anti-cholinergic burden and adverse clinical outcomes. The Annals of Family Medicine, 2017, 15(6): 561-569.

18. Gerretsen P, Pollock B G. Drugs with anticholinergic properties: a current perspective on use and safety. Expert opinion on drug safety, 2011, 10(5): 751-765.

19. Howland R H. Potential Adverse Effects of Discontinuing Psychotropic Drugs--Part 1: Adrenergic, Cholinergic, and Histamine Drugs. Journal of psychosocial nursing and mental health services, 2010, 48(6): 11-14.

20. Giachetti A, Giraldo E, Ladinsky H, et al. Binding and functional profiles of the selective M1 muscarinic receptor antagonists trihexyphenidyl and dicyclomine. British journal of pharmacology, 1986, 89(1): 83-90.

21. Sathienluckana T, Unaharassamee W, Suthisisang C, et al. Anticholinergic discontinuation and cognitive functions in patients with schizophrenia: a pharmacist-physician collaboration in the outpatient department. Integrated pharmacy research \& practice, 2018, 7: 161.

Cite this article as: Charisse C, Claudia L, Tammie L D, Irreversible effects of anticholinergic withdrawal in the elderly: a case report[J]. Aging Pathobiology and Therapeutics, 2021, 3(2): 28-31. 